\title{
The Mobile Mathematics Curriculum-based Measurement Application for Elementary students
}

\author{
Mengping Tsuei \\ Graduate School of Curriculum and Instructional Communications Technology \\ National Taipei University of Education
}

\begin{abstract}
The aim of this study was to develop the application software, mathematics curriculum based measurement (iCBM), for children on the mobile tablet device. The iCBM was developed by various mobile technologies. Two features of iCBM were stated in this paper including the online measurement design and the automatically digital scoring rules. To exam students' perceptions about the use of iCBM system, 34 fifth-grade elementary students participated in the study.

The results of this study indicated that students showed the positive attitudes toward using the iCBM system as well as using mobile tablet devices for taking math tests.
\end{abstract}

\section{Introduction}

Via the mobile tablet devices such as iPad, Eee Pad, children can use their fingers to surfe the websites, typing emails, writing texts, reading books, swiping photos, and switching between various applications (Apps). The touching and writing screen on the tablet technologies have added functionality by using a stylus to write directly on the screen. This feature can help users to make sketches, diagrams and mathematical formulas, in the process of solving problems. Moreover, the light weight of mobile tablets is very suitable for young learners.

The most promising benefit of using technology based assessment systems is the timely and precise information generated for each student's performance in specific content areas [1]. The mobile tablet devices provides a more flexible environment for computer based assessment than paper-and-pencil assessment. As computers continue to penetrate all aspects of education, paper-andpencil assessment becomes disassociated from current learning practices [2]. Previous research have documented the effectiveness of computer-based assessment systems in mathematics by various technologies including diagnosis systems [1], dynamic assessment systems [3], intelligent tutoring systems [4] and adaptive tests [5].

However, there are some limitations of computer based assessment in mathematics for elementary children. First, most of the computer based assessment systems in mathematics are based on the objective tests and closed-type items, like true-false, multiple choice, fill-in-blank questions. Many systems are designed primarily for accessing an individual's mastery of single mathematical concept. These assessment systems can't match the trend in mathematics education which emphasizes the various dimensional aspects of students' math abilities [6]. Recent work in math assessment promotes the use of innovative question-types such as drag-and-drop items and problem-solving questions. This approach reflects a more authentic assessment of examinees' knowledge, skills, and abilities [7]. Therefore, the math question-types provided by the current computer based assessment systems were limited. Second, most applications have limited abilities for measuring young children's mathematics abilities corresponsive to their math curriculum. The results of assessment cannot be used forindexing the student's level of competence in the local school curriculum.

The curriculum-based measurement (CBM) is a databased, problem-solving model for indexing students' academic competence and progress through ongoing assessment [8]. There are several essential features and advantages of the CBM model including (1) the measures are tied to each student's curriculum, (2) the implementing procedures are designed for a short duration (3) the CBM probes are capable of having many multiple forms for repeated measurement, (4) the results are sensitive to the improvement of students' achievements over time [8]. Longitudinal CBM studies have indicated that incorporating CBM feedbacks into instructional planning facilitates general educators to provide more effective instructional programs and thus promote students' achievements in various subject domains, such as reading [9] and mathematics [10].

Computerized CBM system has been developed such as the Monitoring Basic Skills Progress (MBSP) software [11] and the web-based curriculum-based measurement system (ECBM) [12]. The results of these research also indicated that the use of computers helped teachers implement CBM not only saving substantial time in collecting data, but also analysing ongoing assessment information in an effective way [11][12]. However, there is a lack of 
research on developing automated scoring by computer algorithms to provide instant feedbacks in computerized curriculum-based measurement system. As the use of mobile tablet devices in the classroom grows, computer based testing in mathematics for children can be combined with mobile tablet technology and the curriculum-based measurement methods. Therefore, the purposes of this study were:

1. To develop the mobile mathematic curriculumbased measurement application (iCBM) in mobile tablet device for elementary students.

2. To exam students' perceptions about the use of iCBM system.

\section{Related works}

MBSP was developed to collect and manage students' CBM mathematics data. One of the most challenging tasks in CBM mathematics research by using MBSP was the content validity and technical adequacy [13]. The National Council of Teachers of Mathematics' Principle and Standards for School Mathematics emphasizes a student's ability in applying math-related concepts to think and reason mathematically [6]. The MBSP provided two different versions of CBM probes, the basic math computation and the basic math concepts and applications. Every CBM probe in MBSP was designed by one single question-type. The single problem-type CBM probes in MBSP may decrease correlations between CBM and the general measures of mathematics [14]. Moreover, another limitation of MBSP is that the math probes cannot be managed by teachers or administrators. The set of computation included 30 probes at each grade level. Each computation test consisted of 25 math problems, and each concept and application test consisted of 12 math problems.

The ECBM system with dynamic features can generate mixed-types of mathematics probes, track students' progress and provide diagnosed information [12]. Moreover, ECBM provided each teacher with his/her own privilege to dynamically generate CBM probes through module selection. The positive findings suggested that applying class-wide dynamic-growth modeling as well as the assessment of integrated mathematics competency in the instructional processes facilitated students' mathematics learning. However, the math probes generated by the ECBM system were implemented based on the paper-and-pencil test for elementary students. After grading, teachers inputted the scores into the ECBM system. Previous research indicated that the challenge for developing computerized CBM system was the digital scoring process.

The digital scoring process was based on aggregating the correct digits for each question. The correct digit was defined as the right numeral at the right place including the numeral written in reversed form. For a student answering 31 x $5=$ (155) in horizontal type of fill-in-blank question. He or she was awarded 3 correct digits. Another example was asking students to rewrite number sentence 31 × $5=$ (155) as multiplying table as the following, then the correct digit scores are 10.

Apparently, students gained more digits on multistep problems. Every question in CBM probe was not worth equally. The digital scoring process was an effective way for assessing students' math proficiency by giving the full or partial credits. However, the digital scoring process was complicated and time-consuming as reflected by teachers [12]. The automated scoring is needed to facilitate CBM implementations in ongoing assessment for students.

The automated scoring in the computer based assessment was a very promising approach without burdening the teachers [15]. However, this automation of computer based assessment system focused on the objective tests. Previous studies adopted the mathematical expression parser [16] and symbolic computation principles [17] for scoring open-ended mathematics questions. Both of these techniques illustrated the natural languages and artificial intelligence principles, which can be used successfully for scoring open-ended math questions on full- or partial-credited bases. However, the artificial intelligent techniques for system development were time consuming and not able to incorporate various math question-types in elementary curriculum.

Therefore, this study proposed the iCBM system which was developed to address these challenges by incorporating automated scoring schema for measuring students' math proficiency. Students' perceptions about using iCBM system were also presented in this study.

\section{Method}

\subsection{Instruments}

The iCBM was developed on the Android mobile platform with EeePad mobile device to implement CBM for elementary students. The iCBM extended the previous ECBM system by adding the online measurement and automated scoring modules. The Adobe Air, Asp.Net with C\# programming and MS SQL database technologies were adopted in this study. Students can login the ICBM for online CBM testing. The iCBM provided online testing interface with various math question-types in math curriculum in Taiwan. When students finish the CBM test, their scores will be recorded in the database by the automated scoring module of iCBM.

The data for students' perceptions about using iCBM system were collected using a survey comprising on demographics and item scales. 
Cronbach's alpha was used to determine the reliability of the questionnaire. The survey was found to be reliable (Cronbach's alpha $=0.89$ ).

\subsection{Participants}

The pilot evaluation of the iCBM system was conducted in 2012. This preliminary study was based on the fourth-grade CBM probes. However, the fourth-grade students just began to learn these contents for a month when the study implemented. Therefore, the fifth-grade students were selected.

In this study, thirty-four fifth-grade students (14 boys, 20 girls) from two classes in an elementary school in Taipei, Taiwan participated. They were divided by four groups for testing the iCBM system on mobile tablet device. The researcher of this study explained how to use the system. They followed the researcher's instructions to complete one iCBM test. Then, they took one iCBM test individually.

\section{Results}

\subsection{The iCBM system}

The iCBM system architecture was composed two main modules:

1. The online measurement module. The online measurement module was developed for implementing CBM probes on the mobile tablet devices for elementary children. The online testing probes were generated by teachers using the ECBM system. The systematic random selection was adopted in the ECBM system to sample 10 nonrepeated questions. These questions reflect 10 different learning objectives in each semester's curriculum in accord with students' grade level. Therefore, every CBM probe was an alternative form.

Figure 1 presented the iCBM online testing interface. On the top of the screen was the math questions. On the left of the screen was the question number which marked written or not written. The blue background was the canvas area where students can write math expressions on the touch screen by hand. On the bottom screen of ICBM interface was the math keypad. It was developed to improve the input accuracy for elementary students. Students can drag the math keypad to any spot on the screen. There were two math keypads provided by the iCBM (Figure 1). The basic math keypad included numbers (0-9), equation symbols $(+,-, \mathrm{x}, \div)$, and deleting function. The dynamic keypad was shown according to the definitions in each question in the itembank, such as km, gram, degree, square centimeters, and Chinese numerical characters.

To develop the online testing interfaces for various question-types, the third- and fourth-grade math curriculum were analyzed. In the first phase, there were nine question-types developed in the iCBM system for online measurement (Table 1) including true-false choice, multiple choice, matching questions, fill-in-blanks, fill-in-blanks with place values, fill-in-blanks with making sentences, rewriting number sentences as records, computing with records (adding, subtraction, multiplication, division) and word problems with making sentences. The operation questions (i. e. drawing a 3 centimeter radius circle) will be developed in the second phase of this project.

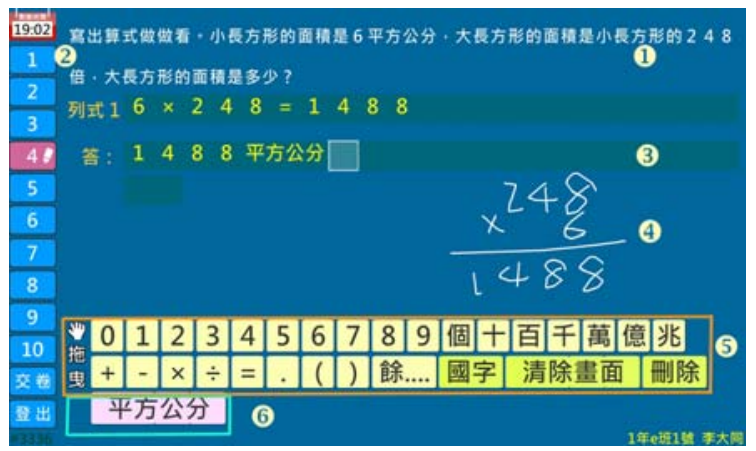

Figure 1. The interface of iCBM (1) question area (2) question number (3) math sentences (4) drawing area (5) basic keypad (6) dynamic keypad

Table 1. The mathematics question-types in the iCBM system

\begin{tabular}{|c|c|c|c|c|c|c|}
\hline Question-types & \multicolumn{6}{|l|}{ Example } \\
\hline True-false choice & \multicolumn{6}{|c|}{$\begin{array}{l}\text { The largest } 7 \text { digit-number is } \\
1000001 \\
\text { True } \\
\text { False }\end{array}$} \\
\hline Multiple choice & \multicolumn{6}{|c|}{$\begin{array}{l}\text { Please choose the following } \\
\text { numbers which is greater than } \\
3 \text { and smaller than } 5 \text {. } \\
\bigcirc \frac{1}{2} \bigcirc 2 \bigcirc 4 \frac{1}{2} \bigcirc 2 \frac{1}{2} \bigcirc 5\end{array}$} \\
\hline Fill-in-blanks & \multicolumn{6}{|c|}{$\begin{array}{l}\text { Write } 74320 \text { in Chinese } \\
\text { characters( }\end{array}$} \\
\hline \multirow[t]{3}{*}{$\begin{array}{l}\text { Fill-in-blanks } \\
\text { with place values }\end{array}$} & \multicolumn{6}{|c|}{$\begin{array}{l}\text { Write the } 47884 \text { in the } \\
\text { following place value }\end{array}$} \\
\hline & \begin{tabular}{|l|}
+ \\
3 \\
位 \\
\end{tabular} & 位 & 位 & 位 & & 無 \\
\hline & table & & & & & \\
\hline $\begin{array}{l}\text { Rewriting number } \\
\text { sentences as } \\
\text { records }\end{array}$ & \multicolumn{6}{|c|}{$\begin{array}{l}\text { Rewriting the following } \\
\text { sentence as records and fill-in- } \\
\text { blank } \\
\left(\begin{array}{ll}(\quad)+35125=50000\end{array}\right.\end{array}$} \\
\hline $\begin{array}{l}\text { Computating with } \\
\text { records }\end{array}$ & \multicolumn{6}{|l|}{$4 0 \longdiv { 8 5 }$} \\
\hline $\begin{array}{l}\text { Fill-in-blanks } \\
\text { with making } \\
\text { sentences }\end{array}$ & \multicolumn{6}{|c|}{$\begin{array}{l}\text { Computing the volume of } \\
\text { following rectangular solid. It } \\
\text { is built by one cubic } \\
\text { centimeter. ( } \quad \text { ) cubic } \\
\text { centimeter }\end{array}$} \\
\hline
\end{tabular}




\begin{tabular}{|l|l|}
\hline $\begin{array}{l}\text { Matching } \\
\text { questions }\end{array}$ & $\begin{array}{l}\text { Please match the items } \\
\text { between left and right items. }\end{array}$ \\
\hline $\begin{array}{l}\text { Word problems } \\
\text { with making } \\
\text { sentences }\end{array}$ & $\begin{array}{l}\text { Please compute with making } \\
\text { sentences. Uncle Lin bought } \\
\text { 3.8 kilogram oranges and 1.25 } \\
\text { kilogram cherry. How many } \\
\text { kilograms did he buy? }\end{array}$ \\
\hline
\end{tabular}

The interface designs for math question-types were developed according to the specific rules defined in the itembank of ECBM system. For example, the question-type of rewriting number sentences as records was defined as the matrix-like data. The width of matrix was the maxim number of digitals before and after the operators (Figure 2). Furthermore, the height of matrix was the number of rows for computation procedures (except the row for results). The question proposed on the Figure 2 was defined as \#\#280 $\times \# \# 39$ and row=2 in the database. The “\#” sign was defined as the blank space in the system. According the rules for the interface design, students can input the answers into the "matrix-like" records on the touch screen.

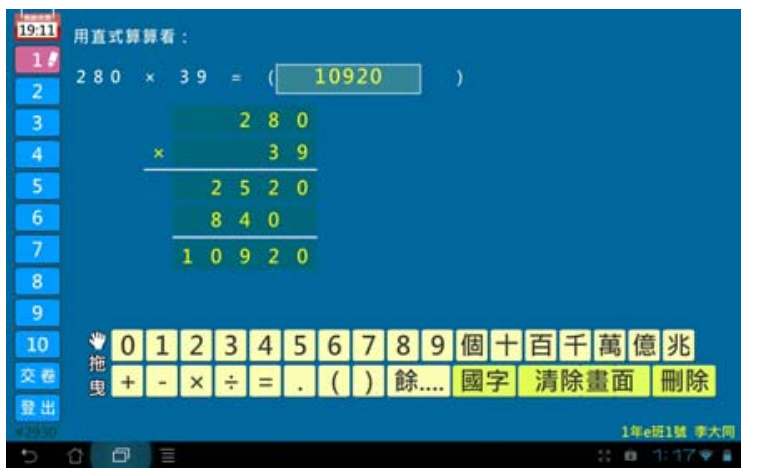

Figure 2. The interface for question-type: Rewriting number sentences as records

Figure presented the interface of another example about the type of matching questions on iCBM (Figure 3). Students were asked to match the equal results between three formulas on the left site and the other three formulas on the right site. The filenames of six images were defined by the question numbers and the place order on the left and right site (i. e. 21754-1, 21754-1r, 21754-2, 21754-2r, 21754-3, 21754-2r). When the system read these pictures, they were easily placed on the left and right by their names. Moreover, these images were developed for the objects which can be pressed to show the connected lines.

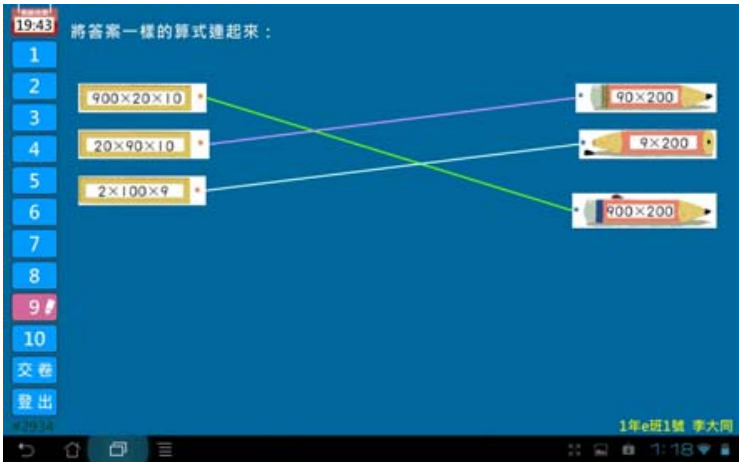

Figure 3. The interface for question-type- Matching question

\section{The automated digital scoring module}

The automatically scoring module of the iCBM system was developed according to the digital scoring rules of CBM model. The digital scoring rules were the methods of accuracy scoring for assessing children's math proficiency [8]. Most of automated scoring algorithms in iCBM were based on matching the answers that students input and the answer field defined in the itembank. For every question, we aggregated the correct digits every part of math question. For the question presented in the figure 2, rewriting number sentences as records 280 x $39=(\quad)$, students have to answer two parts. One is 10920 in the parentheses ( 5 digits), and the other is the multiplication in the records (17 digits). Then, students can get the 22 digits for correct answer. For the above question, the answer field defined in the itembank was [10920][\#\#280, \#\#39, \#2520, \#840\#, 10920]. The brackets were used to separate different part of answers and the "\#” was defined as blank space.

For the question presented in the figure 3 , we defined the [21754-1, 21754-3r][21754-2, 21754$1 \mathrm{r}][21754-3,21754-2 r]$ in the answer fields to specify the corrected pair of objects.

The most complicated automated scoring rule was the question-type of word problems with making sentences and fill-in-blanks with making sentences. Students can input the expressions combining various symbols in the math sentences. Once the expression was entered, the scoring algorithms compared the response to the correct expression. Since expressions have an infinite number of equivalent forms, evaluating equivalency was a key component of these algorithms. The algorithms for scoring word problems in the iCBM were as the following:

Step1: Defining all the final correct answers from the possible expressions into the itembank.

Step2: Computing result of each expression that student entered in the iCBM by the automated scoring parsers.

Step3: Evaluating the corrected expression by the automated scoring parsers. Compare each corrected 
result and the possible answers defined in the itembank. If the corrected results can be found in the itembank, then it is defined as the corrected response. Step4: Aggregating the digits in every correct expression.

Step5: Adding the above digits and the corrected digits in the final answer area by matching rules.

The question presented in the figure 4 was "Teacher Lee bought 240 pencils, she want to give to 32 students evenly. How many pencils can students get? And how many pencils will be left?". The student got total of 17 digits, that was 11 digits for the corrected expression and 6 digits for the final answer area in the figure 4.

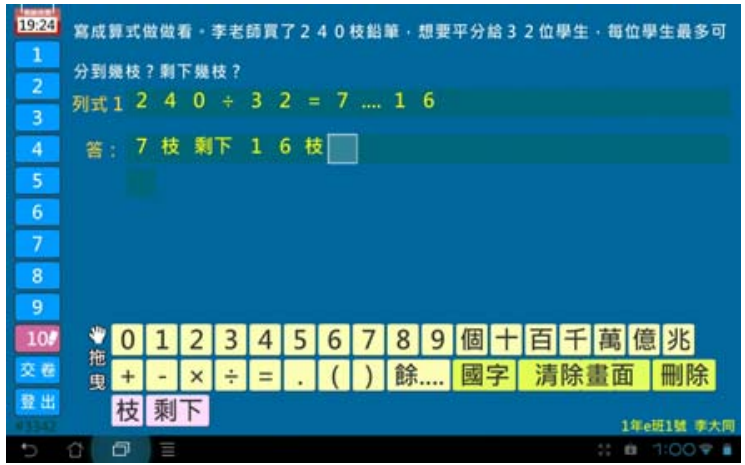

Figure 4. The interface for question-type: Word problems with making sentences

\subsection{Results of pilot evaluation of iCBM system}

The result of average digital scores of students' performance on one of iCBM test were 57.55 (total digital score $=70)(S D=10.06)$. Apparently, the iCBM automatic scoring module performed correctly.

In terms of students' perceptions about using iCBM system, all items of the questionnaire were based on 1-5 point Likert scale, ranging from 1 ("Strongly disagree") to 5 ("Strongly agree").

Table 2 showed descriptive statistics on students' perceptions about using iCBM system. The results of the survey indicated students showed very positive attitude toward using iCBM system on math tests (M $=4.46$, $\mathrm{SD}=0.46)$. The system and interface design were easily for children to use (question 1-3). They also indicated that they like to use iCBM system to take math tests (question 4-7). They also indicated the positive attitude using mobile tablet devices for taking math tests (question 8-15). The interesting finding was that children showed the conservative attitude about the effectiveness of using mobile tablet device and their math performance according to the question 14.

We also found there was no significant difference between genders on the perceptions about using iCBM system $(\mathrm{t}=-7.7, p=0.46)$.
Table 3. The results of students' perceptions about using iCBM system

\begin{tabular}{lrr}
\hline Item & Mean & SD \\
\hline $\begin{array}{l}\text { 1. The image and text are clear to } \\
\text { read. }\end{array}$ & 4.68 & .48 \\
& &
\end{tabular}

2. I can easily operate the iCBM system.

3. There are few errors when I operate $\quad 4.35 \quad .88$ the iCBM system.

4. I think using iCBM system is more $\quad 4.85 \quad 44$ interesting than using paper and pencil on math tests.

5. I hope to use the iCBM system for math tests in our class.

6. I like to use iCBM system on math $\quad 4.79 \quad .54$ tests.

7. I will like mathematics more if I can use iCBM system for math tests.

8. If the teacher let us choose tools for taking math tests (paper-andpencil or mobile tablet device), I will choose the mobile tablet device.

9. I believe that I can learn math better when using the mobile tablet device for math tests.

10. I feel that using mobile tablet device for math test can reduce my test anxiety.

11. I think using mobile tablet device for math tests is easy.

12. I think the mobile tablet device is a useful tool for math tests.

13. Using the iCBM system can help me calculate more quickly.

$4.74 \quad .57$

14. I believe that I can make more progress if I use the mobile table device for math tests.

15. I feel more comfortable when I use the iCBM system to take math tests.

\section{Discussion and conclusions}

Our results indicated that the mobile tablet devices could provide educational opportunities for implementing online mathematics measurement for children. The iCBM system was developed to incorporate math curriculum which providing various interface designs according math questiontypes. According to different question-types, the automated digital scoring module also developed. 
The results of the survey indicated students showed the positive attitudes toward using iCBM system. They satisfied the interface designs and operations of the system. They also showed the positive attitudes toward using mobile tablet devices for math tests.

The iCBM can perform automated digital scoring module correctly. This fact supports our claims about suitability of the mobile tablet platform since it rendered the assessment application friendlier for students. The results were consisted with the previous study showed that students indicated math measurement on the tablet environment is easier than the paper-based exams [2].

Our efforts will concentrate on evaluating the effectiveness and usability of the iCBM system for measuring children' mathematics.

\section{Acknowledgement}

The author thanks the National Science Council of the Republic of China for financially supporting this research under Contract Nos. NSC 100-2410-H152 -014 -MY3.

\section{References}

[1] He, Q., and Tymms, P. (2005) 'A computer-assisted test design and diagnosis system for use by classroom teachers', Journal of Computer Assisted Learning, 21, pp. 419-429.

[2] Siozos, P., Palaigeorgiou, G., Triantafyllakos, G., and Despotakis, T. (2009) 'Computer based testing using digital ink: Participatory design of a tablet PC based assessment application for secondary education', Computer \& Education, pp. 811-819.

[3] Gerber, M. M., Semmel, D. S., and Semmel, M. I. (1994) 'Computer-based dynamic assessment of multidigit Multiplication’, Exceptional Children, 61, pp. 114-125.

[4] Anderson, J. R., Boyle, C. F., and Reiser B. J. (1985) 'Intelligent tutoring systems', Science, 228, pp. 456-462.

[5] Legg S. M., and Buhr, D. C. (1992) 'Computerized adaptive testing with different groups', Educational Measurement: Issues and Practice, 11, pp. 23-27.

[6] National Council of Teachers of Mathematics (2000) Principles and standards for school mathematics, Author, Reston, VA.

[7] Huff, K. L., and Sireci, S. G. (2001) 'Validity issues in computer-based testing', Educational Measurement: Issues and Practice, 20(3), pp. 16-25.

[8] Deno, S. L. (1985) “Curriculum-based measurement: The emerging alternative”, Exceptional Children, 52, pp. 219-232.
[9] Jones, E. D., and Krous, J. P. (1988) 'The effectiveness of databased instruction by student teachers in classrooms for pupils with mild learning handicaps', Teacher Education and Special Education, 11, pp. 9-19.

[10] Stecker P.M. \& Fuchs L.S. (2000) Effecting superior achievement using curriculum-based measurement: the importance of individual progress monitoring. Learning Disabilities Research and Practice, 15, pp. 128-134.

[11] Fuchs, L.S., Hamlett, C.L., and Fuchs D. (1998) Monitoring Basic Skills Progress. Pro-ED, Austin, TX.

[12] Tsuei, M. 'A web-based curriculum-based measurement system for class-wide ongoing assessment', Journal of Computer Assisted Learning, 24, pp, 47-60.

[13] Thurber, R. S., Shinn, M. R., and Smolkowski, K. (2002) 'What is measured in mathematics tests? Construct validity of curriculum-based mathematics measures', School Psychology Review, 31, pp. 498-513.

[14] Helwig, R., Anderson, L., and Tindal, G. (2002) 'Using conceptgrounded, curriculum-based measure in mathematics to predict statewide test scores for middle school students with LD', Journal of Special Education, 36, pp. 102-112.

[15] Masters, J. (2010) 'Automated scoring of an interactive geometry item: A proof-od-concept', The Journal of Technology, Learning, and Assessment, 8(7), pp. 1-38.

[16] Livne, N. L., Livne, O. E., and Wight, C. A. (2007). 'Can automated scoring surpass hand grading of students' constructed responses and error patterns in mathematics?', MERLOT Journal of Online Learning and Teaching, 3(3), 295-306.

[17] Bennett, R. E., Morley, M., and Quardt, D. (2000) 'Three response types for broadening the conception of mathematical problem solving in computerized tests', Applied Psychological Measurement, 24, pp. 294-309. 